\title{
PROPOSTA PARA IMPLANTAÇÃO DE BUSINESS INTELLIGENCE EM SHOPPING CENTER
}

\section{PROPOSAL FOR BUSINESS INTELLIGENCE IMPLEMENTATION IN SHOPPING MALL}

Oswaldo Moreira Pereira; Aglaê Pereira Zaupa; Francisco Virginio Maracci; Caetano Bocchi Pedroso

Universidade do Oeste Paulista - UNOESTE, Faculdade de Informática de Presidente
Prudente, Presidente Prudente, SP
pmoreira.oswaldo@gmail.com; aglae@unoeste.br; francisco@unoeste.br; pedroso@unoeste.br

RESUMO - O presente trabalho tem por objetivo estudar os conceitos envolvidos no processo de Business Intelligence para propor uma solução que apoie a tomada de decisões estratégicas com enfoque na utilização em um shopping center. A tecnologia proposta de solução de $\mathrm{BI}$ utiliza o banco de dados SQL Server e suas ferramentas que permitem a modelagem multidimensional, a transformação de dados em conhecimento estratégico e a apresentação dos resultados por meio do processamento analítico das informações.

Palavras-chave: business intelligence; shopping center; conhecimento estratégico.

ABSTRACT - The aim of the present job was to study the concepts involved in the business intelligence process to propose a solution that supports strategic decision with focus on shopping centers use. The BI technology proposed make use of SQL Server database and its tools that allows the multidimensional modeling, the transformation of data into strategic knowledge and the presentation of the results by the analytical processing information.

Keywords: business intelligence; shopping mall; strategic knowledge.

Recebido em: 14/07/2015

Revisado em: 01/09/2016

Aprovado em: 24/10/2016 


\section{INTRODUÇÃO}

Bussiness Intelligence (BI), é um conjunto de metodologias de gestão implementadas através de ferramentas de software, cuja função é proporcionar ganhos nos processos decisórios gerenciais e da alta administração nas organizações. Esta tecnologia permite às empresas transformar dados, guardados nos seus sistemas, em informações significativas. Permite também aos usuários analisar bases de dados para descobrir informações importantes, ajudando a organização a ter o máximo de exatidão nas tomadas de decisões (PRIMAK, 2008).

Muitos são os conceitos encontrados nas literaturas, porém o mais acessível é o (FREITAS et al., 1997) que define que dado é um elemento da informação (um conjunto de letras ou dígitos) que, tomado isoladamente, não é capaz de transmitir nenhum conhecimento e que um banco de dados é uma coleção de dados relacionados que representa alguns aspectos do mundo real, sendo chamado, às vezes, de minimundo.

Atrelado à definição de dado, encontra-se a informação que é outro elemento que necessita ser conceituado. As informações são o resultado do processamento de dados brutos para revelar seu significado (MACHADO, 2008). O conhecimento é definido como a habilidade de um indivíduo realizar uma tarefa corretamente (FREITAS et al., 1997). Portanto, é possível concluir que dado acrescido de informação e conhecimento possibilita a tomada de decisões, função primordial do BI.

O presente trabalho tem por objetivo estudar os conceitos envolvidos no processo de BI para propor uma solução que apoie a tomada de decisões estratégicas, utilizando o sistema gerenciador de banco de dados SQL Server, com enfoque de sua utilização em um shopping center.

Atualmente, o shopping center, do estudo apresentado, não possui um meio estratégico de tomada de decisões baseado em informações realizando suas ações estratégicas de modo empírico. $O$ intuito do trabalho é propor uma solução de BI adequada que permita realizar decisões de localização de lojas, perfil de clientes, promoções adequadas aos perfis traçados e melhor época para as mesmas. Este tipo de informação é importante para o crescimento estratégico da organização.

Este Artigo apresenta os resultados obtidos em um projeto de iniciação científica do curso superior de Tecnologia em Gestão da Tecnologia da Informação. O mesmo está dividido em seções, organizadas conforme segue: a seção dois apresenta os conceitos fundamentais de $\mathrm{Bl}$; a seção três aborda um dos componentes do $\mathrm{BI}$, o Data Warehouse, 
objeto principal desta pesquisa; a seção quatro descreve a ferramenta de BI Microsoft SQL Server Analysis Services e discorre sobre proposta apresentando o modelo de dados, e ferramentas a serem adotadas.

\section{BUSINESS INTELLIGENCE}

O BI é um termo que define a metodologia do uso de sistemas para obter resultados que facilitem a tomada de decisão nos negócios de uma empresa. Dentre os propósitos do BI está a identificação de padrões nos dados armazenados e, com base nesses padrões, propor análises que possam prever o comportamento de um determinado perfil de cliente, produtos mais vendidos, etc (MACHADO, 2008).

Está cada vez mais difícil tomar decisões por dois motivos: primeiro porque o número de alternativas disponíveis é muito maior do que antes, em virtude de melhores tecnologias e de sistemas de comunicação. Segundo que o custo com erros pode ser alto, em virtude da complexidade e da magnitude das operações da automação e da cadeia de reação que um erro pode causar em muitas partes da organização (TURBAN et al., 2009\}.

O uso do BI minimiza a dificuldade nas tomadas de decisões por ser um conjunto de conceitos e métodos que visa melhorar a capacidade de tomada de decisões, utilizando sistemas baseados em fatos do negócio. $\quad \mathrm{O} \quad \mathrm{BI}$ apresenta inúmeras características onde as principais são: extrair e integrar dados de múltiplas fontes, fazer uso da experiência, analisar dados contextualizados, trabalhar com hipóteses, procurar relações de causa e efeito, transformar os registros obtidos em informação útil para o conhecimento empresarial e aumentar a possibilidade de exatidão na tomada de decisão (Primak, 2008).

A proposta de uma solução para a implantação de Business Intelligence em shopping centers, objetivo deste projeto, será feita com base no uso da tecnologia Data Warehouse.

\section{DATA WAREHOUSE}

O termo Data Warehouse (Armazém de Dados) foi descrito por Inmon (1997), como uma coleção de dados orientada por assuntos, integrada, variante no tempo e não volátil, que tem por objetivo dar suporte aos processos de tomada de decisão. Para que exista o DW é necessária à extração, transformação e carga, contida no conceito de ETL (Extract, Transform and Load) podendo ser originados de uma ou mais bases de dados (PRIMAK, 2008; INMON, 1997).

A extração dos dados que formarão um DW pode ser proveniente de várias fontes internas diferentes (banco de dados de 
sistemas transacionais, arquivos textos, planilhas, etc.) e até mesmo em fontes fora da empresa. Em seguida, é preciso fazer a limpeza e transformação dos dados. Nessa parte são corrigidas algumas imperfeições contidas na base de dados transacional, com o objetivo de fornecer ao usuário dados concisos, não redundantes e com qualidade, permitindo uma análise baseada nos valores mais próximos dos reais. Finalmente, é feito o processo de carga do DW, que normalmente é realizada em um banco de dados temporário.

Um banco de dados transacional, realiza o processo de transações em tempo real, também conhecida como OLTP (Online Transaction Processing). Os dados armazenados provem das tarefas fundamentais do negócio podendo ser constantemente alterados, utilizados por grande parte dos funcionários de uma organização e responsáveis por executar funções predeterminadas (MALLACH, 2000).

As aplicações OLAP são usadas pelos gestores em qualquer nível da organização para Ihes permitir análises comparativas que facilitem a sua tomada de decisões diárias. Armazena dados analíticos necessários para as tomadas de decisões da empresa. Isso pode envolver consultas complexas e que, necessitem de um grande número de registros. Um DW armazena informações históricas de muitos anos e por isso deve ter uma grande capacidade de processamento e armazenamento de dados.

A modelagem multidimensional é uma técnica de concepção e visualização de um modelo de dados e de um conjunto de medidas que descrevem aspectos comuns de negócios. É utilizada especialmente para sumarizar e reestruturar dados e apresentálos em visões que suportem a análise dos valores desses dados. Um modelo multidimensional é composto por três elementos básicos: Fato, Dimensões e medidas. O Fato é uma coleção de dados, composta de dados de medidas e de contexto. Cada Fato representa um item, uma transação ou um evento de negócio e é utilizado para analisar o processo de negócio de uma empresa. É tudo aquilo que reflete a evolução dos negócios do dia a dia de uma organização. A Dimensão são os elementos que participam de um fato, sendo os assuntos do negócio. Podem ser definidas, ainda, como as possíveis formas de visualizar os dados que serão informações. Teoricamente são elas que especificam o que deve conter na resposta de uma solicitação a um relatório, por exemplo, relatório de vendas por mês, relatório de vendas por região, por produtos, etc. Medidas são os atributos numéricos que representam um fato, a performance de um indicador de negócios relativo às dimensões que participam desse fato (MACHADO, 2008). 
Uma das principais funções de uma consulta a um DW é obter informações de dados históricos e analisá-los, se produtivos ou não, a fim de tomar decisões para o desenvolvimento do negócio.

Uma das formas de apresentação de um banco de dados multidimensional é através do modelo estrela. No centro da estrela encontra-se a tabela de fato e, ao seu redor, as dimensões. É um modelo simples e eficiente, caracterizado por possuir uma única tabela de fatos e chaves simples nas tabelas de dimensões. Cada dimensão é representada por uma única tabela (KIMBALL E ROSS, 2002).

O modelo Snowflake pode ser considerado um estrela normalizado, pois emprega uma combinação de normalização da base de dados, para manter a integridade e reduzir os dados armazenados de forma redundante (MACHADO, 2008). Neste modelo, as tabelas dimensionais relacionamse com a tabela de fatos, mas algumas dimensões relacionam-se apenas entre elas, isto ocorre para fins de normalização das tabelas dimensionais, visando diminuir o espaço ocupado por estas tabelas.

A partir do armazenamento dos dados na estrutura dimensional escolhida, o próximo passo é a realização de consultas aos dados. OLAP (Processo Analítico On-line) de acordo com Machado (2008) é um conjunto de ferramentas que possibilitam efetuar a análise de dados multidimensionais. A análise multidimensional representa os dados como dimensões e não como tabelas.

Os resultados dessas análises permitem a descoberta de tendências e cenários positivos para o desenvolvimento do negócio, transformando, os dados de um Data Warehouse em informações estratégicas vistas de diferentes perspectivas e de vários níveis de agregação por intermédio dos cubos. Os cubos são massas de dados que retornam das consultas feitas e permitem por operações OLAP as mudanças para níveis e perspectivas diferentes.

Uma das características que devem estar presentes em ferramentas OLAP é a capacidade de efetuar operações para visualização dos dados do DW. A operação Slice corta o cubo mantendo a mesma perspectiva de visualização dos dados, permitindo que o usuário fixe a apresentação em um determinado detalhe. Dice é a operação que muda a perspectiva de visão, girando o cubo e invertendo uma ou mais dimensões. A operação Drill Down ocorre quando o usuário aumenta o nível de detalhe da informação, diminuindo a granularidade. Pode, ainda, ser entendida como a operação que busca um aprofundamento maior sobre os detalhes nas consultas e tem como elemento principal os atributos das dimensões, que são os responsáveis por agregar e totalizar os resultados das 
consultas. Roll Up é o contrário do Drill Down, ocorre quando o usuário aumenta a granularidade, diminuindo o nível de detalhamento da informação. Drill Across é a operação que ocorre quando o usuário pula um nível intermediário dentro de uma mesma dimensão. Por exemplo, a dimensão tempo é composta por ano, semestre, trimestre, mês e dia. A operação Drill Across é executada quando o usuário passa de ano direto para trimestre ou mês (MACHADO, 2008; PRIMAK, 2008).

\section{DESENVOLVIMENTO}

Nesta seção são apresentados o modelo de dados proposto para o $\mathrm{Bl}$, as ferramentas adotadas pela proposta e a utilização das mesmas para a criação do cubo e a visualização do conhecimento gerado pelas análises. O shopping center do estudo de caso possui lojas de diversos segmentos. O intuito do uso de BI é identificar o perfil de clientes em relação ao segmento de lojas, sexo, estado civil, épocas do ano que mais atrai cada perfil traçado. É de interesse, também, do estabelecimento encontrar o perfil de clientes em relação à localização das lojas nos pisos para melhor realizar a alocação de futuras das lojas e maximizar os lucros visto que o valor de aluguel de um espaço é calculado segundo o tamanho do local, mas também segundo o faturamento do lojista. portanto, saber em qual piso uma loja deve ficar para maximizar faturamento do lojista é um questionamento relevante para o estabelecimento.

Atualmente, o estabelecimento utilizase de um software que colhe alguns dados dos consumidores das lojas pelo cadastro das notas fiscais em campanhas promocionais e a partir do levantamento destes dados foi possível realizar a proposta da modelagem de dados que será apresentada posteriormente na seção 5.2. Por questões de sigilo foi vetada a divulgação do nome do estabelecimento estudo desta proposta.

\subsection{MICROSOFT SQL SERVER ANALYSIS SERVICE}

O SQL Server é uma plataforma abrangente de banco de dados que fornece recursos de gerenciamento de dados de classe empresarial com ferramentas de BI integradas das quais o Analysis Services faz parte. A Microsoft Analysis Services é uma ferramenta de Business Intelligence que oferece um conjunto de serviços usados para gerenciar dados em um Data Warehouse ou Data Mart. A conexão é feita via ODBC e permite fontes de dados em diversos formatos.

O SSAS (SQL Server - Analysis Services) cria bases de dados e vários elementos são necessários para que isso ocorra, os primordiais são: Data Source, Data Source View, Dimensões e Cubos. O Uso desses componentes, que são criados manualmente, 
atravéz dos wizards de auxílio permitem que os dados sejam extraídos do DW e organizados em um cubo multidimensional para que as análises dos dados sejam feitas de forma detalhada, utilizando as consultas que o cubo propõem (ZARE et al., 2009).

\subsection{MODELAGEM DE DADOS}

A partir da necessidades levantadas as seguintes tabelas foram definidas: cliente, tempo, lojas e vendas. As tabelas foram formadas a partir da análise realizada in-loco no shopping center, Figura 1.

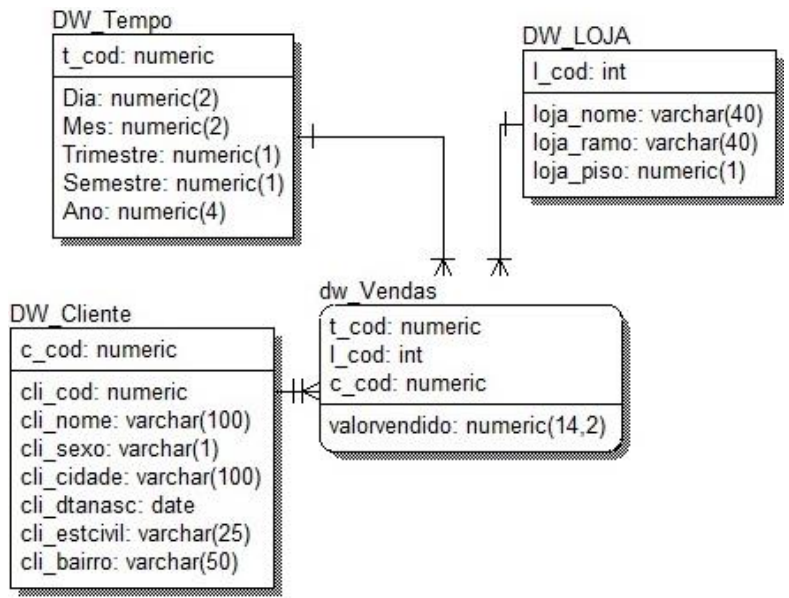

Figura 1. Modelo Proposto.

Fonte: autoria própria.

A estrutura escolhida para organização dos dados deste cubo, foi o modelo dimensional estrela. Esse modelo foi escolhido por apresentar várias vantagens que foram fundamentais para sua escolha (KIMBALL E ROSS; 2002): (1) modelo de arquitetura padrão e previsível, ferramentas de consulta e interfaces do usuário podem se valer disso para fazer suas interfaces mais amigáveis e fazer um processamento mais eficiente, (3) todas as dimensões do modelo são equivalentes, ou seja, podem ser vistas como pontos de entrada simétricos para a tabela de fatos, (4) as interfaces do usuário são simétricas, as estratégias de consulta são simétricas, e o SQL gerado, baseado no modelo, é simétrico, (5) modelo dimensional é totalmente flexível para suportar a inclusão de novos elementos de dados, bem como mudanças que ocorram no projeto, (6) todas as tabelas de fato e dimensão podem ser alteradas simplesmente acrescentando novas colunas à tabela. Nenhuma ferramenta de consulta ou relatório precisa ser alterada de forma a acomodar as mudanças.

\subsection{CRIANDO O CUBO}

Para a criação de um cubo, é necessário que exista um Data Warehouse povoado, portanto é necessário que o tomador de decisões do negócio em questão, no caso o shopping center, disponibilize os dados que compõe suas bases de dados.

Para elaboração dessa proposta, o shopping center deve disponibilizar uma tabela única, como na Tabela_Geral da Figura 2 , com os dados necessários para realização do processo de ETL. Após isso, a transformação é realizada e o resultado (Tabela_NOVA_GERAL) utilizado para a carga, conforme a Figura 2. A carga dos dados no DW proposto foi realizada com a utilização 
da ferramenta Import and Export do próprio Micrsoft SQL Server. A Figura 3, ilustra o processo de recebimento dos dados, a extração, transformação e carga no DW para posterior criação do cubo.

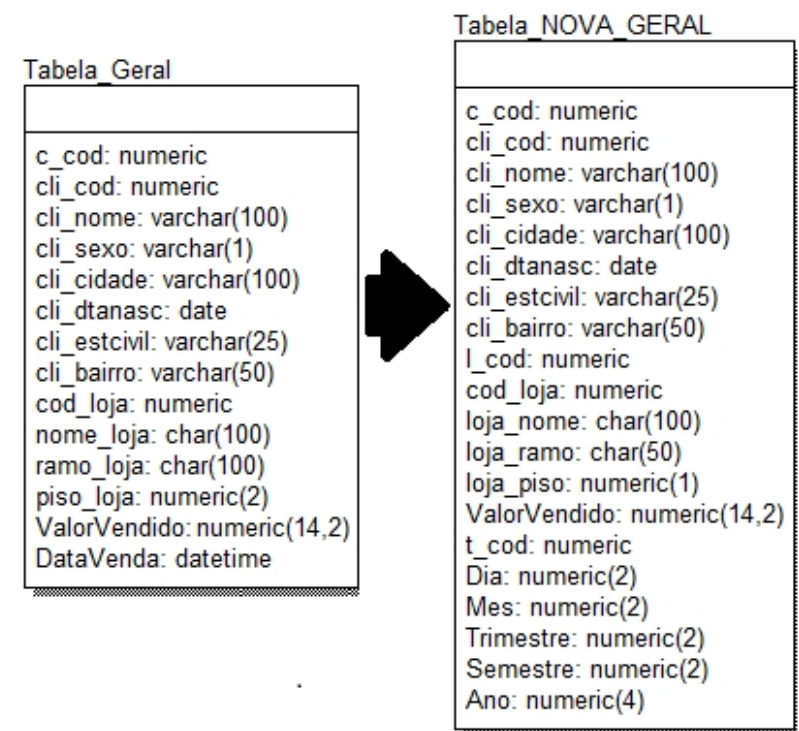

Figura 2. Tabela Recebida e tabela transformada.

Fonte: autoria própria.

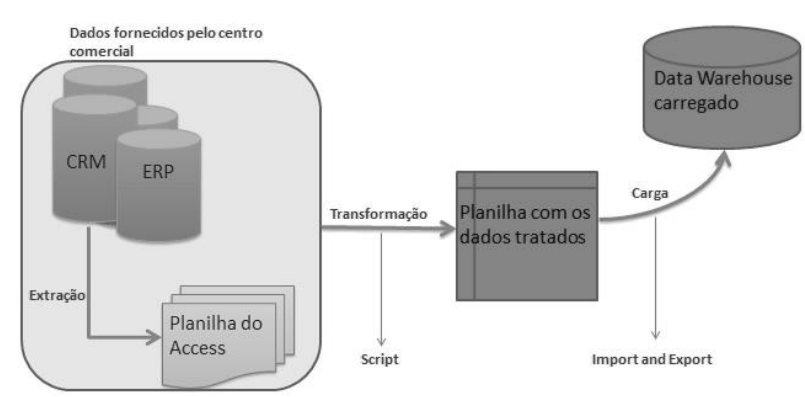

Figura 3. Processo de ETL dos dados proposto.

Fonte: autoria própria.

A partir da carga do DW haverá a criação da solução OLAP. Com o Business Intelligence Development Studio aberto, pertencente ao Microsoft Visual Studio, deve ser criado um novo projeto selecionando o tipo de solução como ilustrado na Figura 4.

Após criado o Analysis Service Project, o próximo passo é criar os componentes dessa solução OLAP. Utilizando a aba Solution Explorer é possível gerenciar as seguintes propriedades destes componentes: (1) Data Source, são os diferentes locais de onde os dados podem ser coletados para as possíveis consultas e análises dos dados; (2) Data Source View, este componente organiza os Data Sources que estão sendo utilizadas para visualização; (3) Cube, é o objeto onde são realizadas as consultas sendo que ao criar o cubo, deve ser selecionado, também, o grupo de medidas que ele usará, esse grupo será potencialmente, a tabela fato da sua modelagem; e (4) Dimension, são as tabelas que foram criadas na modelagem de dados, tabelas que através de suas chaves primárias, compõem a tabela fato.

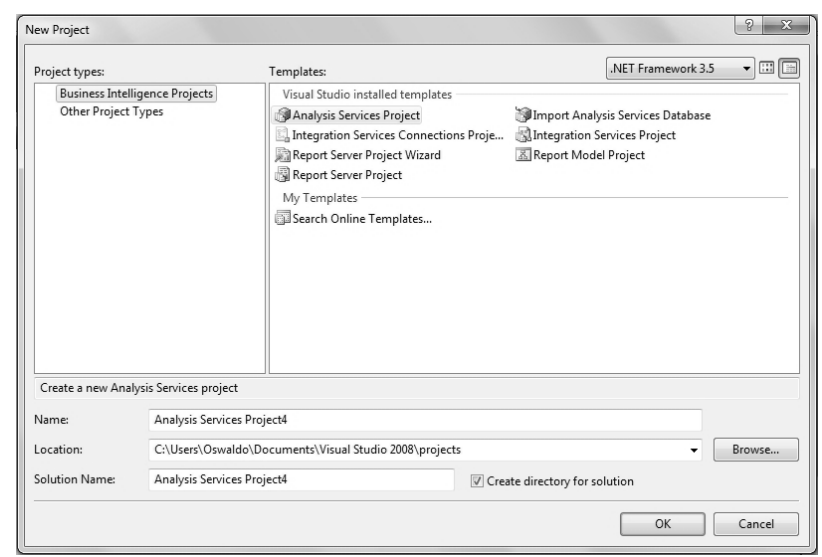

Figura 4. Selecionando o projeto.

Fonte: autoria própria.

Outros componentes, também, podem compor uma solução OLAP, tudo depende da necessidade do usuário, das formas com que acessará o cubo e quais resultados são esperados das consultas que serão feitas. 
Na figura 5, pode-se observar a janela Solution Explorer já com os componentes criados. Uma vez que todos os elementos foram criados e configurados é necessário que este cubo seja implantado, realizando o deploy do cubo que envia o mesmo para a base de dados do Analysis Services. Após isto, o cubo deve ser processado, para que os dados do banco de dados criados anteriormente sejam carregados. Para isso, clica-se com o botão direito do mouse sob a figura que ilustra o projeto no Solution Explorer na opção process, e a Figura 6 aparecerá. Em seguida clique em Run para confirmar o processamento do cubo.

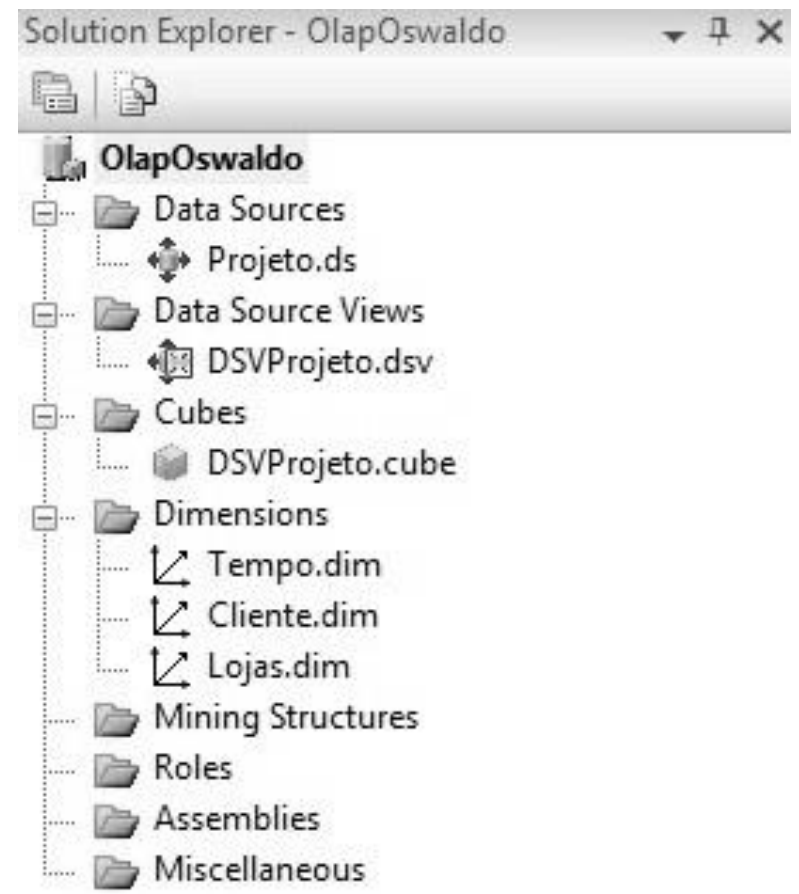

Figura 5. Componentes OLAP do Solution Explorer.

Fonte: autoria própria.

Com o cubo e suas propriedades criadas, é necessário escolher uma ferramenta para a visualização dos dados do cubo através das consultas que podem ser realizadas. Essa rotina de processar o cubo deve ser realizada sempre que ocorrer alguma mudança relativa ao acesso aos dados no Data Warehouse, sempre que os dados no DW forem alterados ou alguma rotina de atualização das tabelas ocorrer.

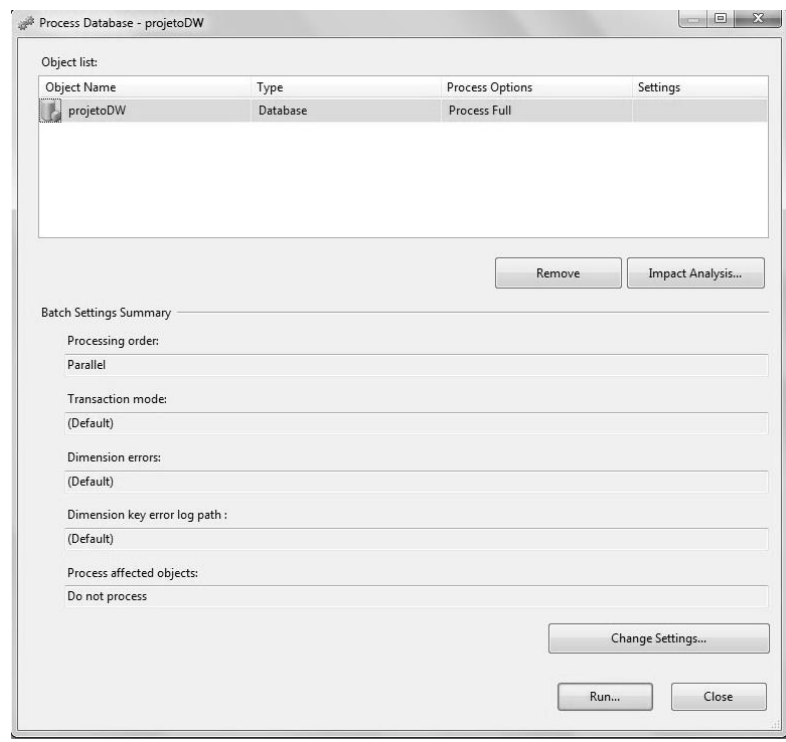

Figura 6. Processando o Cubo.

Fonte: autoria própria.

\subsection{VISUALIZANDO OS DADOS DO CUBO}

Nesta seção são apresentadas duas formas para a visualização do cubo: Microsoft Visual Studio 2008 e Microsoft Excel.

A visualização dos dados no cubo deve ocorrer necessariamente depois que o cubo foi processado como ilustrado na Figura 7. Com o cubo já processado, é necessário clicar na aba Browse do Visual Studio 2008. Ao ser carregada a tela, as dimensões e as medidas do cubo gerado aparecerão no canto esquerdo da tela. Para visualizar os dados que compõem essas dimensões e medidas, é necessário selecionar a hierarquia ou o dado 
para análise e arrastar para o centro da tela, onde serão exibidos no formato de linhas e colunas, que podem ser alteradas de acordo com a necessidade do usuário.

Para a realização das operações OLAP, é necessário que existam hierarquias pré- definidas entre os dados do DW, essas hierarquias podem ser criadas e alteradas de acordo com a necessidade do usuário do cubo e são elas que definem a disposição dos possíveis dados a serem consultados no cubo.

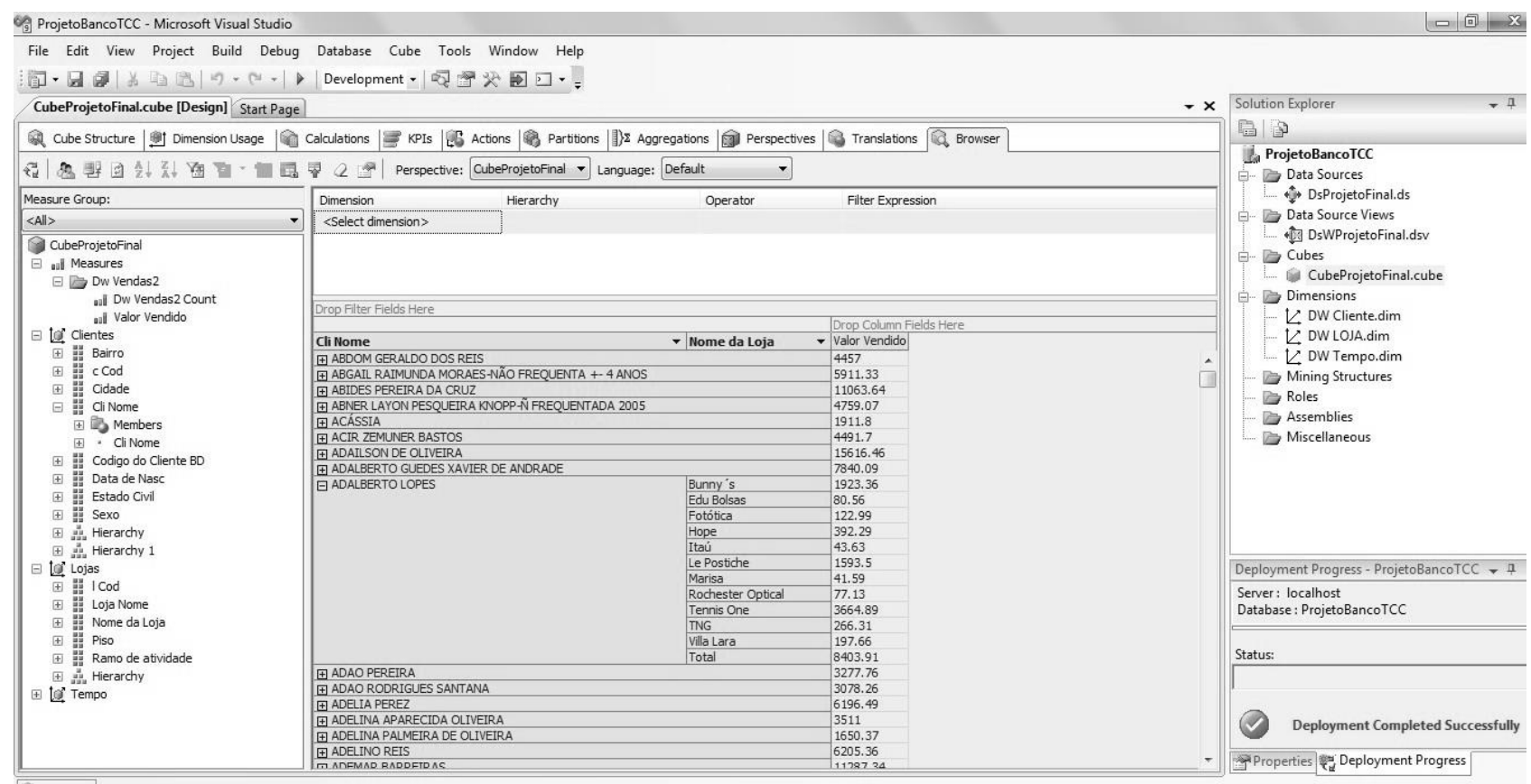

Figura 7. Visualizando dados no Visual Studio 2008. Fonte: autoria própria.

Da mesma forma como os dados foram visualizados ao serem arrastados para o centro da tela, como mostra a Figura 7, para a realização das operações OLAP, é necessário que o usuário identifique a consulta que deseja realizar e as arraste até o centro da tela, modelando as colunas e as linhas de forma agradável para a visualização dos dados resultantes da consulta.

As possíveis consultas a serem feitas são: drill down, drill up, drill across, slice e dice.

A Figura 8 ilustra um Drill Down no cubo desenvolvido neste projeto, onde o valor total vendido é mostrado inicialmente apenas nos anos de 2009 e 2010 e em seguida a consulta se torna mais detalhada e mostra também os semestres do ano em questão.

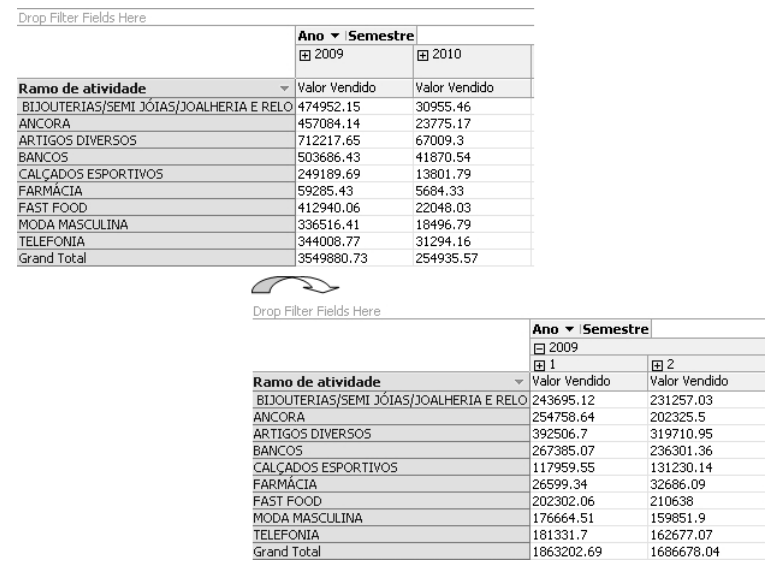

Figura 8. Resultado do Drill Down.

Fonte: autoria própria. 
Outra forma de visualização do cubo é a utilização do Microsoft Excel 2010. O Excel realiza a conexão com o DW e exibe os resultados de consultas de forma similar às exibidas com o uso do Visual Studio. Para realizar a visualização do cubo através do Microsoft Excel, com o software aberto, o usuário seleciona a aba dados e em seguida a opção de outras fontes, várias opções serão apresentadas, o usuário deve escolher a segunda opção, dos serviços de Análise. Em seguida basta indicar o nome do servidor e o tipo de autenticação que ele tem, e selecionar o cubo que será analisado, como ilustram as Figuras 9 e 10 respectivamente.

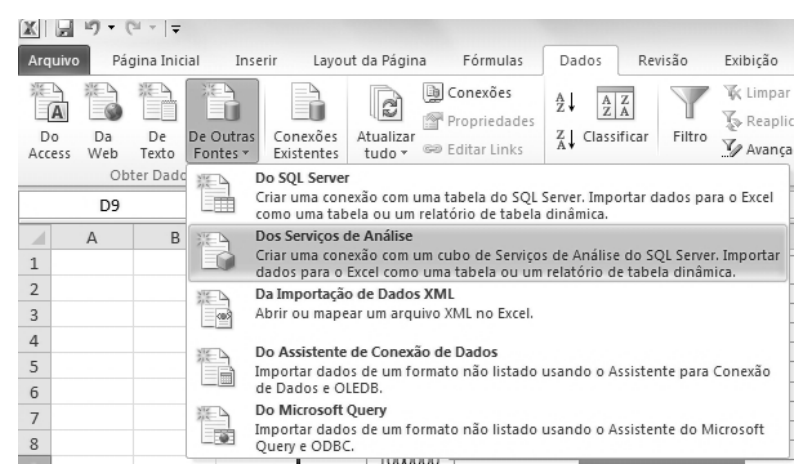

Figura 9. Conectando ao cubo com o

Excel.

Fonte: autoria própria.

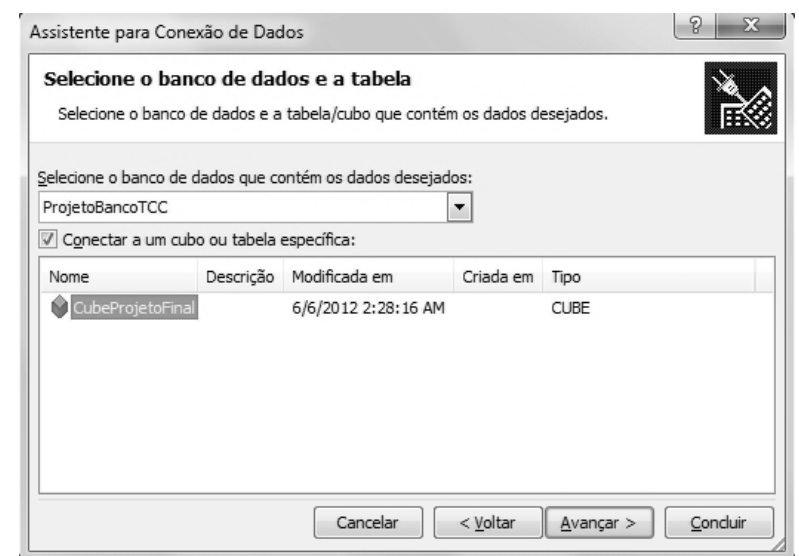

Figura 20. Janela de seleção do cubo.

Fonte: autoria própria.
Dentre as características encontradas no Excel, o que se destaca são os filtros e a possibilidade de analisar simultaneamente dados de fontes diferentes e, gerar gráficos que facilitam a comparação para a tomada de decisões. Por se tratar de uma ferramenta mais conhecida, se comparado ao Visual Studio, o Excel se torna mais prático para analisar os dados do cubo. Quando existe alguma dúvida quanto ao seu uso, é possível encontrar as soluções de forma mais rápida por ser uma ferramenta em português, ao contrário do Visual Studio que é totalmente em inglês.

Para realizar as operações OLAP com o Excel, o usuário deve selecionar os itens que irão compor as linhas e colunas da consulta em questão, por meio da janela lista de campos da tabela dinâmica, como ilustra a Figura 11. Enquanto o usuário seleciona os dados que devem ser analisados o gráfico correspondente aos dados selecionados é automaticamente exibido, bem como os relatórios com os números da consulta. 


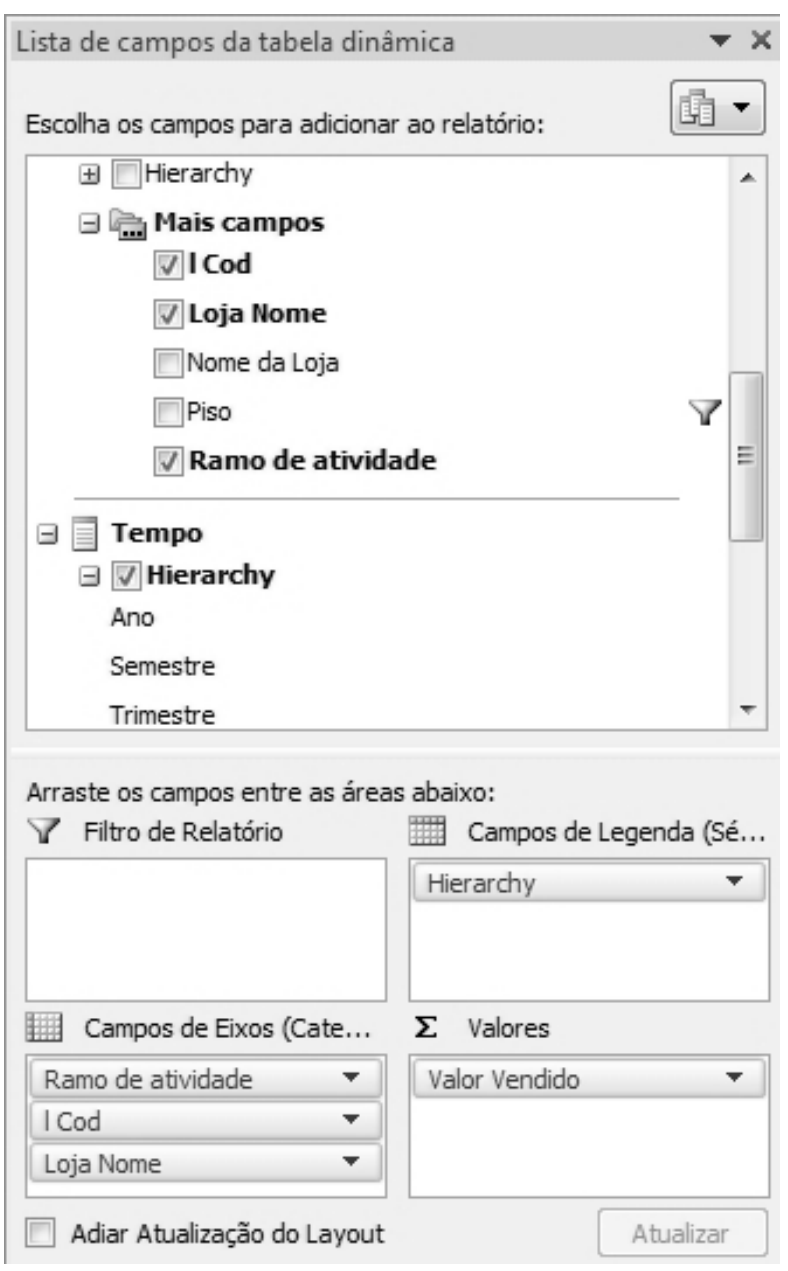

Figura 11. Janela de seleção dos dados para consulta.

Fonte: autoria própria.

A Figura 12 ilustra a operação OLAP Slice, operação que corta o cubo, isto é, a visualização sob a perspectiva de uma maior quantidade de dados para menos dados, nesse caso o total vendido no primeiro e no segundo andar do shopping center e na sequência, os dados apenas do primeiro andar.

\begin{tabular}{|c|c|c|c|c|}
\hline \multirow{2}{*}{$\begin{array}{l}\text { ValorVentido } \\
\text { Bítulos de linha }\end{array}$} & \multicolumn{3}{|c|}{ Rietulos de Colina, $\bar{I}$} & \multirow[b]{2}{*}{ Tobalgeal } \\
\hline & 71 & 2 & 2 & \\
\hline \multicolumn{2}{|c|}{ 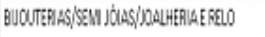 } & 117698,67 & 17976879 & 22445,45 \\
\hline \multicolumn{2}{|l|}{ ANDSPA } & $2 \$ 1,1,1$ & 59241,13 & 330359,14 \\
\hline \multicolumn{2}{|l|}{ APTOOSDNGRBSOS } & 2444,16 & 20391,4 & $451: 3,58$ \\
\hline \multicolumn{2}{|l|}{ suncos } & 315235,53 & & $315: 55,53$ \\
\hline \multicolumn{2}{|c|}{ 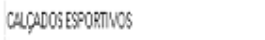 } & & 16279,9 & 2. 1627996 \\
\hline
\end{tabular}

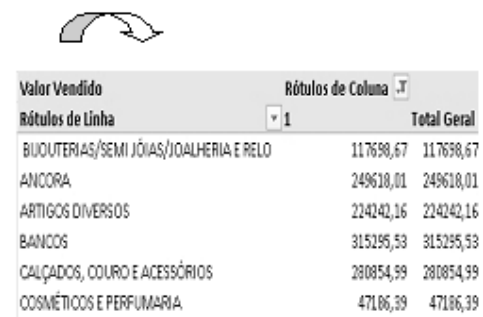

Figura 32. Resultado da operação OLAP Slice no Excel.

Fonte: autoria própria.

Muitas são as ferramentas para visualização e realização de consultas em um cubo. Este trabalho usa como referências as duas mais conhecidas no mercado de $\mathrm{BI}$, o Visual Studio e o Microsoft Excel, ambos têm características parecidas para visualização do cubo, porém o Microsoft Excel se destaca por ser mais fácil de usar, estar em português e dispor de elementos como gráficos e filtros que não são encontrados com facilidade no Visual Studio, que por sua vez, manipula a estrutura do cubo de forma excepcional. Para o usuário final, o tomador de decisão da empresa, o Excel é a melhor opção.

\section{CONSIDERAÇÕES FINAIS}

As mudanças nos modelos de negócio que vem acontecendo ao longo dos tempos, têm norteado analistas, desenvolvedores e gestores a buscarem novas soluções de respostas aos problemas encontrados no enredo dos negócios. Uma solução já 
utilizada por muitas organizações e ainda em crescimento é o uso do $\mathrm{BI}$.

Com o uso do $\mathrm{BI}$, mais especificamente de um cubo multidimensional, a análise dos dados existentes no DW, garante ao tomador de decisões estratégicas de negócio repostas mais rápidas que podem levá-lo à ascendência nas vendas, mais satisfação do cliente, mais comodidade para o cliente e sobre tudo reduzir a possibilidade de erros em vários aspectos que possam ser analisados com os dados que estejam no DW.

O uso de uma ferramenta de $\mathrm{Bl}$ possibilita maior valor às informações necessárias para tomada de decisões. A maneira mais crucial de se diferenciar uma empresa de sua concorrência é fazer um trabalho destacado com a informação.

Uma das maiores dificuldades para a elaboração da solução proposta neste trabalho foi o acesso aos dados da empresa do estudo de caso, pois tais dados são cruciais para o sucesso da empresa e sigilosos, dificultando o acesso ao mesmo e a obrigatoriedade da não divulgação dos mesmos.

O shopping center analisado terceiriza a sua base de dados, e muitas decisões referentes à disposição das lojas, ou ao comportamento dos clientes são tomadas mediante respostas de pesquisas realizadas manualmente por funcionários da empresa in-loco nas lojas, periodicamente. Outra fonte de informações é o cadastro das notas fiscais para participação em promoções feitas pelo shopping center.

A proposta de uma solução para implantação de $\mathrm{BI}$ no shopping center em questão pôde ser verificada como positiva, visto que as necessidades de respostas rápidas e objetivas podem ser sanadas com o uso da solução proposta, o que não era possível sem o uso de uma ferramenta que fizesse a transformação de dados em conhecimento estratégico e a apresentação dos resultados por meio do processamento analítico das informações.

A proposta foi apresentada para a administração do shopping center e responsáveis do departamento de TI como forma de avaliação obtendo uma avaliação positiva. A administração demonstrou interesse em realizar melhorias na proposta apresentada para a futura implantação da solução.

Sendo assim, o aperfeiçoamento da modelagem de dados proposta pela solução e a implantação da solução obtida com a pesquisa, são propostas de trabalhos futuros.

Portanto, conclui-se que shopping centers são empresas a serem exploradas para a elaboração de soluções de BI para o crescimento dos negócios, e que essa é uma solução viável e eficaz para o local utilizado como objeto do estudo de caso. Com o uso dessa solução é possível acabar com a 
lentidão no processo de coleta de dados e geração de conhecimento estratégico.

\section{REFERÊNCIAS}

FREITAS, $H$. et al. Informação e decisão: sistemas de apoio e seu impacto. 1. ed. Porto Alegre: Ortiz, 1997.

INMON, W. H. Como construir o Data Warehouse. 1. ed. Rio de Janeiro: Campus, 1997.

KIMBALL, R.; ROSS, M. The data warehouse toolkit: the complete guide to dimensional modeling. 2. ed. Canadá: John Wiley Consumer, 2002.

MACHADO, F. R. N. Tecnologia e Projeto de Data Warehouse: uma visão multidimensional. 4. ed. São Paulo: Érica, 2008.

MALLACH, E. G. Decision support and data warehouse systems. 1. ed. New York: Mcgraw-Hill, 2000.

PRIMAK, F. V. Decisões com B.I. (Business Intelligence). 1. ed. Rio de Janeiro: Ciência Moderna, 2008.

TURBAN, E. et al. Business Intelligence: um enfoque gerencial para a inteligência do negócio. 1. ed. São Paulo: Bookman, 2009.

ZARE, R. et al. Professional Microsoft SQL Server Analysis Serrvice. 1. ed. Canadá: John Wiley Consumer, 2009. 